\title{
Happy families: a twin study of humour
}

\author{
Lynn Cherkas, Fran Hochberg, Alex J MacGregor, Harold Snieder and Tim D Spector
}

Twin Research and Genetic Epidemiology Unit, St Thomas' Hospital, London, UK

\begin{abstract}
The objective of this study was to estimate how much of an individual's appreciation of humour is influenced by genetic factors, the shared environment or the individual's unique environment. A population-based classical twin study of 127 pairs of female twins (71 monozygous (MZ) and 56 dizygous (DZ) pairs) aged 20-75 from the St Thomas' UK Adult Twin Registry elicited responses to five 'Far Side' Larson cartoons on a scale of $0-10$. Within both MZ and DZ twin pairs, the tetrachoric correlations of responses to all five cartoons were significantly greater than zero. Furthermore, the correlations for $M Z$ and $D Z$ twins were of similar magnitude and in some cases the DZ correlation was greater than that of the $M Z$ twins. This pattern of correlations suggests that shared environment rather then genetic effects contributes to cartoon appreciation. Multivariate model-fitting confirmed that these data were best explained by a model that allowed for the contribution of the shared environment and random environmental factors, but not genetic effects. However, there did not appear to be a general humour factor underlying responses to all five cartoons and no effect of age was seen. The shared environment, rather than genetic factors, explains the familial aggregation of humour appreciation as assessed by the specific 'off the wall' cognitive type of cartoons used in this study. Twin Research (2000) 3, 17-22.
\end{abstract}

Keywords: genetics, humour, twins, multivariate, model fitting

\section{Introduction}

'What is your response to cartoons? Would other members of your family respond in a similar way, or acquaintances with similar educational or socioeconomic backgrounds? Do you think that your response is unique?

These questions refer to three factors that have been identified as influences on human behavioural and physiological development: genetics, shared family environment and the individual's unique environment. All can be assessed directly in a study of $M Z$ and $D Z$ twins. ${ }^{1}$

Twins, unless separated at birth or during their youth, grow up in a shared environment. Often twins continue to have similar lifestyles, even after they have left this shared environment. Because of their differential genetic similarities - MZ (monozygous) twins share $100 \%$ of their genes and DZ (dizygous) twins share on average $50 \%$, ie no more than do ordinary siblings - twins provide a sound model by which to estimate the relative importance of genetic and environmental influences in a controlled manner.

The origins of humour have been the source of much speculation, ${ }^{2-7}$ but have never been subject to examination in a twin study. There is no accepted or

Correspondence: Dr Tim Spector, Twin Research and Genetic Epidemiology Unit, St Thomas' Hospital, Lambeth Palace Road, London SE1 7EH, UK. E-mail: tim.spector@kcl.ac.uk

Received 14 January 1999; revised 25 March 1999; accepted 10 August 1999 standardised way of testing humour. In this report we present the results of a study of the responses of $M Z$ and DZ twins to five 'Far Side' Larson ${ }^{8}$ cartoons which represent one facet of humour. We use this information to quantify the relative importance of genetic, shared environmental and unique environmental effects on appreciation of this specific type of humour.

\section{Methods \\ Participants}

The subjects were 127 pairs of female-female twins, selected at random from the St Thomas' Adult UK Twin Registry, ${ }^{9}$ which comprises a cohort of MZ and DZ female twins pairs. All are healthy volunteers who were originally recruited through a national media campaign and from twin registers. ${ }^{10}$ The zygosity of the twins was measured by questionnaire ${ }^{11}$ and validated by multiplex DNA fingerprinting using variable tandem repeats.

\section{Cartoon selection}

In choosing cartoons for this study, we have taken into account Eysenck's observations on the subject of humour. ${ }^{2}$ Eysenck classified humour appreciation into three main types; conative, affective and cognitive, as represented in Figure2. The conative aspect rel ates to the satisfaction of the desire for superiority, the 'joyful consciousness of superior adaptation' (eg 
gloating over physical imperfections or accidents). Affective jokes address the emotions and rely on various cultural assumptions, such as sexual, racial, or ethnic themes to derive their humour (eg 'dirty' jokes or of the Englishman, the Irishman and the Scotsman type). With cognitive jokes, appreciation 'results from the sudden, insightful integration of contradictory or incongruous ideas, attitudes, or sentiments which are experienced objectively', that is, from 'getting the joke'. Eysenck further argues that the conative and affective aspects of humour can be grouped together under the general name of 'orectic' (from the Greek orektikos yearning after, to reach after), as they are related much more closely to each other than either is to the cognitive aspect.

Because of their nature, orectic jokes may alienate various members of the audience and thus potentially bias the sample. To avoid this, we intentionally used only 'clever' jokes involving predominantly the cognitive aspect of humour, as there is no reason to expect biased responses 'when the stimulus or theme is of a less affective nature'. ${ }^{3}$

Five cartoons (of which two are reproduced in Figure 1) were selected for a humour questionnaire

\section{THE FAR SIDE}

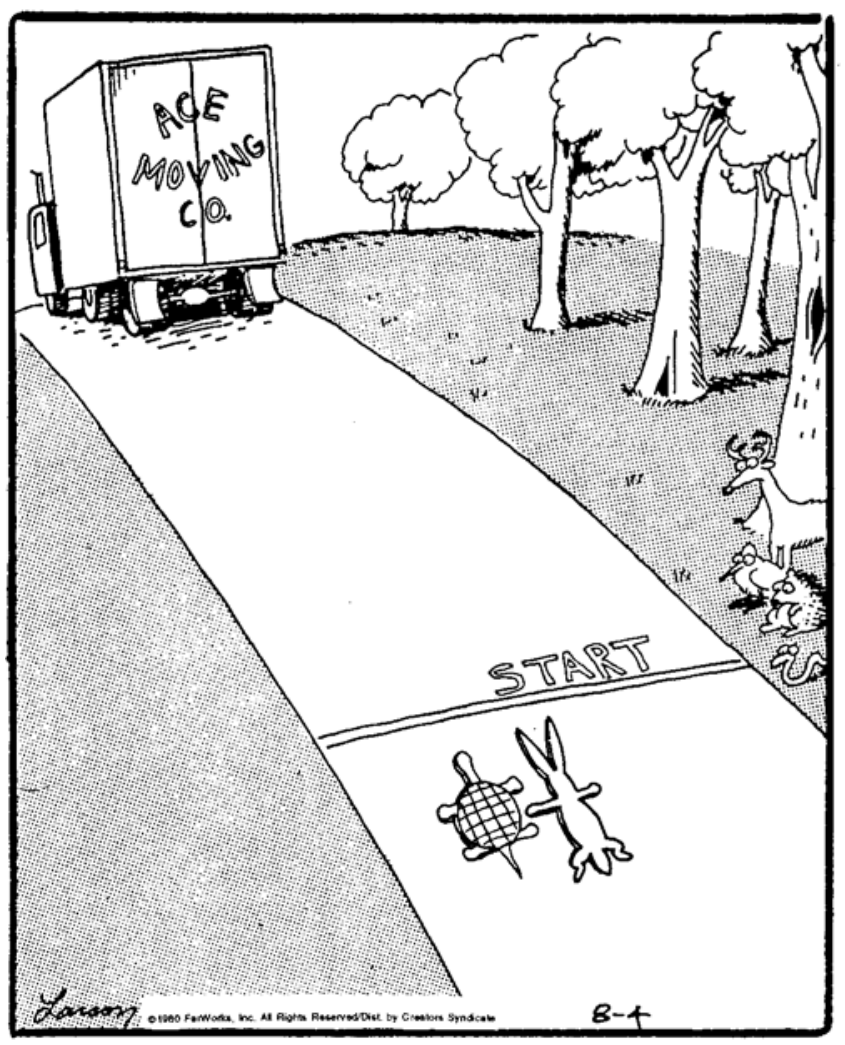

The Far Side by Gary Larson (1) 1980 FarWorks, Inc. Used with permission.

from a group of 15 cartoons given as a pilot study to 30 members of the Twin Research and Genetic Epidemiology Unit. All 15 cartoons were drawn by Gary Larson and had not been seen previously by members of the unit. They were taken from A Prehistory of the Far Side: A 10th Anniversary Exhibit. ${ }^{8}$ The five cartoons chosen were those that had elicited the broadest range of responses in the pilot study. All were images, although one (Cartoon 2) had an accompanying caption.

Cartoon 1: a dog tethered to the mast of a Viking ship wags its tail as its owner and other Vikings return from a burning and presumably pillaged castle.

Cartoon 2: a group of people stands in a doorway to a room in which a composer sits at his piano. The composer's head is slumped against the keyboard and his arm, or the skel etal remains thereof, hangs to his side. The caption below reads 'shhhh!...the Maestro is decomposing!'

Cartoon 3: a woman stands in her living room, peering into a fishbowl; her eye magnified and

THE FAR SIDE

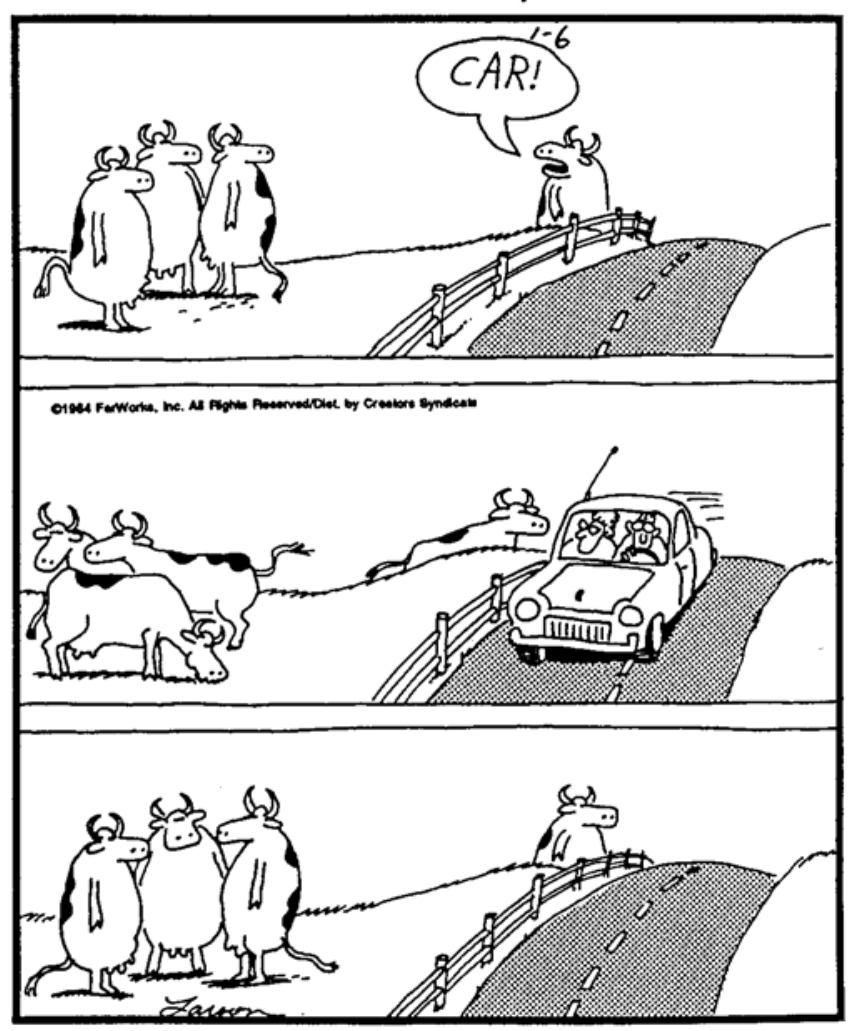

The Far Side by Gary Larson (1) 1984 FarWorks, Inc. Used with permission All Rights Reserved

Figure 1 Examples of the cartoons (4 and 5 ) shown to the participants 
distorted by the water. Meanwhile, an enormous eye fills the window of the room, staring at her.

Cartoons 4 and 5: see Figure 1.

\section{Cartoon scoring}

Both members of a twin pair were given the selfcompletion humour questionnaire in a controlled setting, during the course of the general twin research visit. The questionnaire, comprising the five cartoons, had written instructions asking them to rate the cartoons on a scale of zero to ten, where 0 meant that the cartoon was 'a waste of paper' and 10 meant that it was 'one of the funniest cartoons they had ever seen'. This was similar to a previously validated 7-point scale. ${ }^{12}$ Both twins filled in the questionnaire at the same time in adjoining rooms, so they were unable to comment on the exercise, nor were they able to look at their respective twin's reactions or responses to the cartoons. The twins were given 5 minutes for this task.

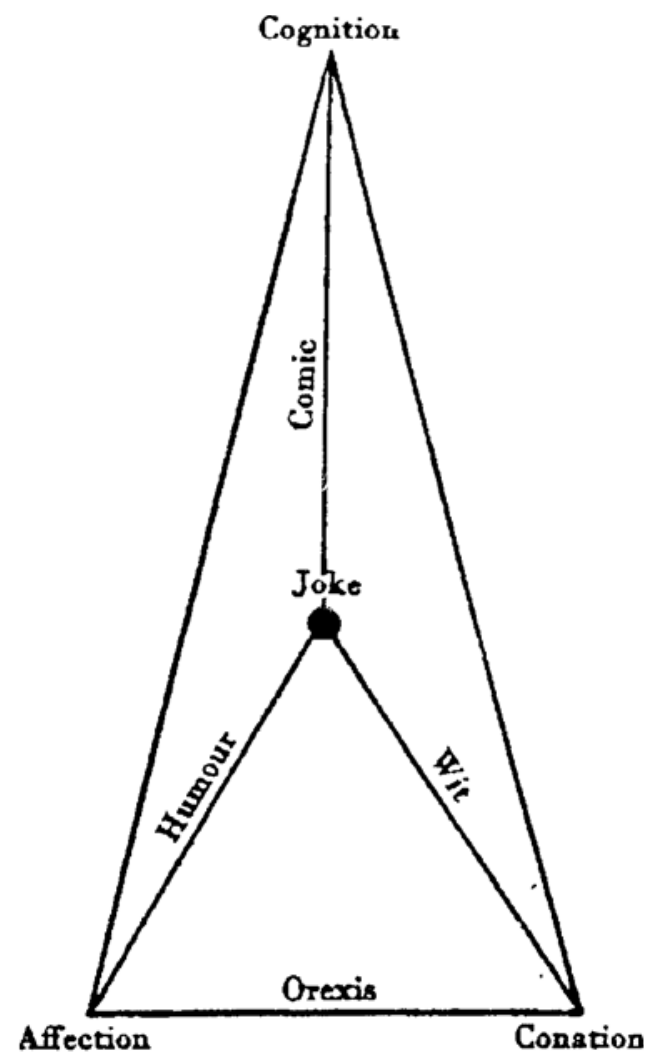

Figure2 This diagram, taken from Eysenck ${ }^{2}$, represents the structure of the joke, showing the three-fold determination of laughter by cognitive, conative and affective factors

\section{Analysis}

The responses to each cartoon were scored as categorical variables and tetrachoric correlations were derived of pairwise $M Z$ and $D Z$ twin similarities for these responses, using PRELIS. ${ }^{13}$ In order to separate genetic from environmental effects, a multivariate model-fitting approach was used. This enabled all the information in the phenotypic polychoric correlation (and associated asymptotic covariance matrices) of the responses to all five cartoons for both $M Z$ and $D Z$ twin pairs to be anal ysed simultaneously. Three multivariate models were tested: the Cholesky decomposition, independent pathway and common pathway models. ${ }^{1}$ Whilst all these models decompose the variance into three components of variation: $A$ (the additive genetic), $C$ (the shared environmental) and $E$ (the individual's unique experiences), each represents different ways in which genes and the environment may affect the observed correlations between the outcome measures (ie the responses to the five cartoons).

The Cholesky decomposition model allowed us to explore the possible existence of factors (genetic and environmental) that are shared by the outcome measures. Thus the model implies a first common genetic factor that loads on all five response measures (A1), a second common genetic factor (A2) that loads on all but the first measure, a third common genetic factor (A3) that loads on all but the first two measures etc. Common shared environmental factors (C) and unique individual environmental factors (E) load on the response measures in a similar pattern to those of the genetic factors described. The independent pathway model is a sub-model of the Cholesky, assuming only one common factor of each type $(A, C$ and $E$ ) loading on all the outcome measures. Beside these three common factors, each of the five response measures is associated with three independent $A, C$ and $E$ factors specific to that response. In the common pathway model, both genes and the environment are assumed to contribute to a single latent (unmeasured) variable eg 'sense of humour', which is responsible for the observed correlation of the response scores. Genetic and environmental factors specific to each response are al so incorporated in the model.

The purpose of the model-fitting procedure is to explain the pattern of observed correlations using as few parameters as possible. Models were fitted to the correlation and associated asymptotic weight matrices by the method of weighted least squares using $\mathrm{Mx} .{ }^{14} \mathrm{Mx}$ provides parameter estimates, a chi-square test $\left(\chi^{2}\right)$ of the goodness of fit of the model, and the Akaike's information criterion (AIC). The overall $\chi^{2}$ test measures the agreement between the observed and predicted variances and covariances in the 
different zygosity groups. Sub-models were compared by hierarchic $\chi^{2}$ tests, in which the $\chi^{2}$ value for a nested model is subtracted from that of the full model. The degrees of freedom for this test are equal to the difference between the degrees of freedom for the full and the nested model. The fit of the model is evaluated by Akaike's information criterion, calculated as $\chi^{2}-2 \mathrm{df}$. The model with the lowest AIC reflects the best balance of goodness of fit and simplicity of the model. ${ }^{1}$ In this best-fitting model, the proportion of variance explained by the specific variance components influencing each cartoon is estimated.

Preliminary statistical analysis was done in STATA, ${ }^{15}$ the polychoric correlation and associated asymptotic covariance (weight) matrices were cal culated using PRELIS. ${ }^{13}$

\section{Results}

The means and tetrachoric correlations of the responses to each cartoon for $M Z$ and $D Z$ twin pairs are shown in Table 1. MZ twins had a mean age of 60 years $(S D=11 \mathrm{yrs}$, range $20-75 \mathrm{yrs}$ ). DZ twins had a mean age of 52 years (SD $=12 \mathrm{yrs}$, range 24-71 yrs). Twin pairs showed considerable similarity in their responses to all five cartoons, with correlations ranging from 0.24 to 0.61 . The correlation of age with the five individual response scores was $0.18,0.15,-0.07,-0.06$ and -0.03 . As the amount of variation accounted for by age was at most $3 \%$, age was not included in the models. The lack of excess correlation in MZ when compared to DZ pairs indicates that shared environmental factors (which might include, for example, the family environment, peer influences and education) rather than genetic influences contribute to the similarity in responses to the cartoons.

Model-fitting to the polychoric correlation and asymptotic covariance matrices of responses confirmed that a model without genetic factors and containing parameters for shared and unique environment only provided the best explanation of the data within each of the multivariate models tested

Table $1 \quad M Z$ and DZ means and tetrachoric correlations for individual cartoons

\begin{tabular}{lllllll}
\hline & \multicolumn{3}{c}{$M Z(n=71$ pairs $)$} & \multicolumn{3}{c}{ DZ $(n=56$ pairs $)$} \\
& Mean & S.D. & Corr & Mean & S.D. & Corr \\
\hline Cartoon 1 & 2.10 & 2.14 & 0.28 & 2.01 & 2.36 & 0.32 \\
Cartoon 2 & 5.09 & 2.68 & 0.38 & 4.48 & 2.76 & 0.45 \\
Cartoon 3 & 2.81 & 2.39 & 0.32 & 2.73 & 2.59 & 0.24 \\
Cartoon 4 & 4.03 & 2.89 & 0.39 & 3.59 & 2.72 & 0.61 \\
Cartoon 5 & 4.37 & 2.73 & 0.50 & 4.52 & 2.67 & 0.41 \\
\hline
\end{tabular}

SD: standard deviation of mean; Corr: tetrachoric correlations
Table 2 Results of model fitting to cartoon responses

\begin{tabular}{|c|c|c|c|c|c|c|}
\hline Model & $\chi^{2}$ & $\mathrm{df}$ & $\mathrm{AIC}$ & $\Delta \chi^{2}$ & $\Delta \mathrm{df}$ & $\mathrm{P}$ \\
\hline \multicolumn{7}{|c|}{ Cholesky model } \\
\hline ACE & 127.93 & 50 & 27.93 & & & \\
\hline $\mathrm{AE}$ & 234.00 & 65 & 104.25 & 106.07 & 15 & $<0.001^{a}$ \\
\hline CE & 134.35 & 65 & 4.35 & 6.42 & 15 & $\mathrm{~ns}^{\mathrm{a}}$ \\
\hline \multicolumn{7}{|c|}{ Independent pathway model } \\
\hline ACE & 161.02 & 65 & 31.02 & & & \\
\hline $\mathrm{AE}$ & 335.30 & 75 & 185.31 & 174.28 & 10 & $<0.001^{b}$ \\
\hline CE & 177.52 & 75 & 27.52 & 16.50 & 10 & $<0.10^{\mathrm{b}}$ \\
\hline \multicolumn{7}{|c|}{ Common pathway model } \\
\hline ACE & 226.07 & 73 & 80.07 & & & \\
\hline $\mathrm{AE}$ & 391.20 & 79 & 233.20 & 165.13 & 6 & $<0.001^{\mathrm{c}}$ \\
\hline CE & 230.84 & 79 & 72.84 & 4.77 & 6 & $n s^{c}$ \\
\hline
\end{tabular}

acompared with ACE model; ${ }^{b}$ compared with ACE model; 'compared with ACE model; $\chi^{2}: \chi^{2}$ goodness of fit stastistic; df: degrees of freedom; $\Delta \chi^{2}$ : difference in $\chi^{2} ; \Delta$ df: difference in degrees of freedom. AIC: Akaike's information criterion $\left(A I C=\chi^{2}-2 d f\right)$ used to evaluate the fit of the models. Best fitting model for each of the three types of multivariate models is in bold

(Table2). Genetic factors offered no significant contribution under any of the three models. The Cholesky decomposition pathway model offered the best fitting CE model overall (by AIC), suggesting that the environmental covariation between the response measures cannot be adequately explained by one common shared (or unique) environmental factor loading on the responses to all five cartoons (independent pathway model) nor can the covariation be explained by a single phenotypic latent variable 'humour' (common pathway model).

The parameter estimates and 95\% confidence intervals for the best fitting Cholesky CE model are given in Table3. The contribution of the shared environment to variation in responses to the five cartoons varies between $37 \%$ and $59 \%$. Non-shared individual environmental effects account for the remaining proportions of the phenotypic variance.

\section{Discussion}

For many dimensions of human behaviour, including personality and social attitudes a significant genetic contribution has been reported. ${ }^{16}$ Sense of

Table 3 Estimated variance components from best-fitting CE Cholesky model with $95 \%$ lower and upper confidence intervals

\begin{tabular}{lll}
\hline & $\begin{array}{l}\text { Shared- } \\
\text { environmental } \\
\text { variance C }(95 \% \mathrm{Cl})\end{array}$ & $\begin{array}{l}\text { Non-shared } \\
\text { environmental } \\
\text { variance E }(95 \% \mathrm{Cl})\end{array}$ \\
\hline Cartoon 1 & $37 \%(26-47 \%)$ & $63 \%(43-84 \%)$ \\
Cartoon 2 & $48 \%(41-55 \%)$ & $52 \%(33-71 \%)$ \\
Cartoon 3 & $35 \%(25-44 \%)$ & $65 \%(45-85 \%)$ \\
Cartoon 4 & $59 \%(52-67 \%)$ & $41 \%(22-60 \%)$ \\
Cartoon 5 & $49 \%(40-57 \%)$ & $51 \%(32-71 \%)$ \\
\hline
\end{tabular}


humour, however, until now has not been independently investigated in these terms, although links between sex, age, personality and intelligence with different aspects of humour have been studied. ${ }^{2-7}$ There is evidence that humour appreciation varies with age and conservatism, depending on the humour content; that extroverts and males are more likely to appreciate orectic type jokes and that the magnitude of response to a cognitive joke depends on the match between the 'cognitive demand' of the stimulus and the individual's psychometric abilities. The lack of a correlation with age in the present study is surprising but may reflect the specific content of the cartoons.

Just as the relative contribution of genetic and environmental influences to sense of humour is largely irresolute within the literature, sense of humour itself is ill defined. Sense of humour commonly refers at once to the ability to respond to events, scenarios, or cultural productions which have been termed funny within a given culture (ie appreciation of humour) and conversely, to the ability to create, or draw attention to these same events, scenarios, or productions (ie creation of humour). Furthermore, humour appreciation cannot necessarily be equated with laughter. ${ }^{4}$ As for the determinants of humour, the general conclusions seem to be that 'there are no clear objective criteria for determining what is funny and what is not ${ }^{3}$ and that 'it is something either innate or closely related to personality'. ${ }^{4}$

If humour is innate, then we might expect to find it is strongly influenced by genetic factors. The postulated link between humour and personality al so suggests there may be a role for genetic influences, as researchers have al ready demonstrated a genetic contribution to many aspects of personal ity. ${ }^{16}$ Furthermore, appreciation of a cognitive type joke may be associated with $I Q,{ }^{5}$ which is generally accepted to have some degree of genetic determination. ${ }^{17}$ Unfortunately, we did not have any cognitive information on the twins in this study, so could not investigate any relationship between their responses and psychometric abilities. However, given these well recognised associations, it is surprising that our results do not support the notion of a genetic contribution to the appreciation of humour.

The reason we did not detect a genetic component may relate to the problem of definition. This study did not seek to define sense of humour, a task which no philosopher, psychologist, or scientist has yet fulfilled, though many have tried, from Aristotle and Cicero to Schopenhauer, Kant, and Freud. ${ }^{2}$ We were interested only in approximating the aetiology of appreciation of cognitive humour, being just one aspect of sense of humour defined by Eysenck. ${ }^{2}$ Thus, our choice of material may have influenced our results. Appreciation of visual cartoons of this 'off-beat' nature may well have a different aetiology to the appreciation of jokes which represent more closely the conative or affective aspects of humour defined by Eysenck. Determinants of humour creation may al so vary.

Nonethel ess, our data do suggest that appreciation or not of a Larson-type cartoon in the cognitive domain is largely influenced by the shared environment, with no significant contribution from genetic factors. It is interesting to speculate over the relative impact of, for example, family, peers, teachers, religion, the media and politics in this regard. Humour has long been theorised by psychologists and anthropologists al ike to be a means of expressing what is most utterly human, yet it seems from our findings that at least some aspects of humour can be learned.

\section{Acknowledgements}

We are grateful for the help of the Twin Research interviewers Lucy Campbell, Ursula Perks, Karen Smith, Naomi Welsh and Lucy Wormald and to Olivia Schiffer and Dr Chris Hammond for administering the questionnaires. We are also grateful to the twins themselves for their willing participation in the study and their sense of humour. This research was partly funded by grants from the Chronic Disease Research Foundation, The Wellcome Trust and Gemini Research Ltd.

\section{References}

1 Neale MC, Cardon LR. Methodology for Genetic Studies of Twins and Families. Kluwer: Dordrecht, 1992.

2 Eysenck HJ. The appreciation of humour: an experimental and theoretical study. Br J Psychol 1942; 32: 295-309.

3 Brodzinsky DM, Rubien J. Humor production as a function of sex of subject, creativity, and cartoon content. J Consult Clin Psychol 1976; 44: 597-600.

4 Thorson JA, Powell FC. Sense of humor and dimensions of personality. J Clin Psychol 1993; 44: 799-809.

5 Thorson JA, Powell FC. Development and validation of a multidimensional sense of humor scale. J Clin Psychol 1993; 49: 13-23.

6 Schaier AH, Cicerelli VG. Age differences in humor comprehension and appreciation in old age. J Gerontol 1976; 31: $577-582$.

7 Ruch W, McGhee PE, HehI F. Age differences in the enjoyment of incongruity-resolution and nonsense humour during adulthood. Psychol Aging 1990; 5(3): 348-355.

8 Larson G. A Prehistory of the Far Side: A 10th Anniversary Exhibit. Creators Syndicate International, 1992.

9 St Thomas' Adult UK Twin Registry. Twin Res 1998; 1(1): 47.

10 Spector TD, Cicuttini F, Baker J, Loughlin J, Hart D. Genetic influences on osteoarthritis in women: a twin study. Br Med J 1996: 312: 940-944. 
11 Martin NG, Martin PG. The inheritance of scholastic abilities in a sample of twins. Ascertainment of the sample and diagnosis of zygosity. Ann Hum Genet 1975; 39: 213-218.

12 Johnson AM. Language ability and sex affect humour appreciation. Percept Motor Skills 1992; 75: 571-581.

13 Joreskog KG, Sorbom D. PRELIS: a preprocessor for LISREL. 2nd edn. Scientific Software: Mooresville, IN, 1988.

14 Neale MC. Mx: Statistical Modelling, 2nd edn. Department of Psychiatry, University of Virginia: Richmond, 1995.
15 StataCorp. Stata statistical software, release 5.0. Stata Corp: College Station, 1997.

16 Eaves LJ, Eysenck HJ, Martin NG. Genes, Culture, and Personality: An Empirical Approach. Academic Press: London, 1989.

17 Henderson ND. Human behavior genetics. Ann Rev Psychol 1982; 33: 403-440. 